This is the accepted version of the article published by Taylor \& Francis: Leonardo M. Elias and Juan E. Martínez-Legaz. A generalization of the strong Fitzpatrick inequality, Optimization Vol. 66, Iss. 6, 2017. The final version is available at: $\mathrm{https}: / /$ doi.org/10.1080/02331934.2016.1179738

\title{
A generalization of the strong Fitzpatrick inequality
}

\author{
Leonardo M. Elias* \\ Programa de Pós-Graduação em Matemática \\ Universidade Federal do Paraná \\ CP 19081, 81531-980 Curitiba, PR, Brazil \\ Juan E. Martínez-Legaz \\ Departament d'Economia i d'Història Econòmica \\ Universitat Autònoma de Barcelona \\ 08193 Bellaterra, Spain \\ and \\ BGSMath, Barcelona, Spain
}

\begin{abstract}
We present a generalization of the strong Fitzpatrick inequality in the context of reflexive Banach spaces, involving a twisted bigger conjugate function. We also introduce a related family of gap functions for maximal monotone inclusion problems.
\end{abstract}

Keywords: Strong Fitzpatrick inequality; gap functions, maximal monotone operators; twisted bigger conjugate functions; Tykhonov well-posed functions

\section{Introduction}

The main result of this mote is a generalization of the strong Fitzpatrick inequality ([8, Theorem 4], [4, Theorem 9.7.2]) in the context of reflexive Banach spaces. In our generalized inequality, a twisted bigger conjugate function $[7$, Definition 19.14] defined on the product of the space with its dual plays the role that the norm on this product plays in the case of the classical strong inequality. Related to the new inequality, we introduce a new family of gap functions, parameterized by Tykhonov well-posed twisted bigger conjugate functions, for

${ }^{*}$ This research was supported by CAPES, under grant BEX 3843/14-9. This author is affiliated to CAPES Foundation, Ministry of Education of Brazil, Brasília - DF 70040-020, Brazil.

${ }^{\dagger}$ This author was supported by the MINECO of Spain, Grant MTM2014-59179-C2-2-P, and under Australian Research Council's Discovery Projects funding scheme (project number DP140103213). He is affiliated to MOVE (Markets, Organizations and Votes in Economics). 
a general maximal monotone inclusion problem, that is, the problem of finding a zero of a maximal monotone operator. By means of a gap function one can reformulate the maximal monotone inclusion problem as a convex optimization problem. We compare our new family with a gap function recently proposed in $[2]$.

The rest of the paper is organized as follows. Section 2 deals with the fundamental notions on maximal monotone operators and Fitzpatrick functions that are used in the paper. In Section 3 we present our generalization of the strong Fitzpatrick inequality. Section 4 introduces a related family of gap functions for maximal monotone inclusion problems.

\section{Preliminaries}

Let $(X,\|\cdot\|)$ be a real Banach space with dual $\left(X^{*},\|\cdot\|_{*}\right)$. We will denote by $\langle\cdot, \cdot\rangle: X \times X^{*} \rightarrow \mathbb{R}$ the duality pairing between these spaces. The duality pairing between the space $X \times X^{*}$ and $X^{*} \times X$ is the function on $\left(X \times X^{*}\right) \times X^{*} \times X$, also denoted by $\langle\cdot, \cdot\rangle$, given by

$$
\left\langle\left(x, x^{*}\right),\left(y^{*}, y\right)\right\rangle=\left\langle x, y^{*}\right\rangle+\left\langle y, x^{*}\right\rangle .
$$

We recall that $X^{*} \times X$ can be canonically identified with a subspace of the dual space $\left(X \times X^{*}\right)^{*}$ of $X \times X^{*}$; under this identification, one has $\left(X \times X^{*}\right)^{*}=$ $X^{*} \times X$ if and only if the space is reflexive.

Let $T: X \rightrightarrows X^{*}$ be a set-valued operator. The graph and the domain of $T$ are given, respectively, by

$$
\operatorname{gph} T:=\left\{\left(x, x^{*}\right) \in X \times X^{*} \mid x^{*} \in T(x)\right\}
$$

and

$$
\operatorname{dom} T:=\{x \in X \mid T(x) \neq \emptyset\} .
$$

Recall that $T$ is said to be monotone if

$$
\left\langle x-y, x^{*}-y^{*}\right\rangle \geq 0
$$

whenever $x, y \in X$ and $\left(x, x^{*}\right),\left(y, y^{*}\right) \in g p h T$.

A monotone map $T: X \rightrightarrows X^{*}$ is said to be maximal monotone if there is no monotone map whose graph properly contains the graph of $T$. The Fitzpatrick function associated with a maximal monotone operator $T$ is the lsc convex extended real valued function on $X \times X^{*}$ defined by

$$
F_{T}\left(x, x^{*}\right):=\sup _{\left(y, y^{*}\right) \in g p h T}\left\{\left\langle x-y, y^{*}\right\rangle+\left\langle y, x^{*}\right\rangle\right\} .
$$

Equivalently,

$$
F_{T}\left(x, x^{*}\right)=\left\langle x, x^{*}\right\rangle-\inf _{\left(y, y^{*}\right) \in g p h T}\left\langle x-y, x^{*}-y^{*}\right\rangle .
$$


Since $T$ is maximal monotone, we can easily see that the Fitzpatrick function satisfies

$$
F_{T}\left(x, x^{*}\right) \geq\left\langle x, x^{*}\right\rangle \text { for all }\left(x, x^{*}\right) \in X \times X^{*},
$$

with equality if and only if $\left(x, x^{*}\right) \in g p h T$.

Given an lsc proper convex function $f: X \rightarrow \mathbb{R} \cup\{+\infty\}$, its Fenchel conjugate $f^{*}: X^{*} \rightarrow \mathbb{R} \cup\{+\infty\}$ is defined by

$$
f^{*}\left(x^{*}\right):=\sup _{x^{*} \in X^{*}}\left\{\left\langle x, x^{*}\right\rangle-f^{*}\left(x^{*}\right)\right\} .
$$

Therefore, in view of (1), the restriction of the Fenchel-Moreau conjugate of $g: X \times X^{*} \rightarrow \mathbb{R} \cup\{+\infty\}$ to $X^{*} \times X$ is given by

$$
g^{*}\left(x^{*}, x\right):=\sup _{\left(y, y^{*}\right) \in X \times X^{*}}\left\{\left\langle\left(y, y^{*}\right),\left(x^{*}, x\right)\right\rangle-g\left(y, y^{*}\right)\right\} .
$$

It is not difficult to see that the conjugate $F_{T}^{*}$ of the Fitzpatrick function $F_{T}$ associated with a maximal monotone operator $T$ satisfies

$$
F_{T}^{*}\left(x^{*}, x\right) \geq F_{T}\left(x, x^{*}\right) \text { for all }\left(x, x^{*}\right) \in X \times X^{*} .
$$

Theorem 1 (see [4, Exercise 9.23]) Let $T: X \rightrightarrows X^{*}$ be maximal monotone. Then

$$
F_{T}\left(x, x^{*}\right)-\left\langle x, x^{*}\right\rangle+F_{T}\left(w, w^{*}\right)-\left\langle w, w^{*}\right\rangle \geq-\frac{1}{2}\left\langle x-w, x^{*}-w^{*}\right\rangle
$$

for all $\left(x, x^{*}\right),\left(w, w^{*}\right) \in X \times X^{*}$.

Proof. Using the convexity of the Fitzpatrick function and the fact that it is bounded below by the duality product, we obtain

$$
\begin{aligned}
F_{T}\left(x, x^{*}\right) & -\left\langle x, x^{*}\right\rangle+F_{T}\left(w, w^{*}\right)-\left\langle w, w^{*}\right\rangle \\
& =2\left(\frac{1}{2} F_{T}\left(x, x^{*}\right)+\frac{1}{2} F_{T}\left(w, w^{*}\right)\right)-\left\langle x, x^{*}\right\rangle-\left\langle w, w^{*}\right\rangle \\
& =2\left(\frac{1}{2} F_{T}\left(x, x^{*}\right)+\frac{1}{2} F_{T}\left(w, w^{*}\right)\right)-\left\langle x, x^{*}\right\rangle-\left\langle w, w^{*}\right\rangle \\
& \geq 2 F_{T}\left(\frac{1}{2}(x+w), \frac{1}{2}\left(x^{*}+w^{*}\right)\right)-\left\langle x, x^{*}\right\rangle-\left\langle w, w^{*}\right\rangle \\
& =-\frac{1}{2}\left\langle x-w, x^{*}-w^{*}\right\rangle .
\end{aligned}
$$

We conclude this section enunciating a version of Fenchel Duality Theorem. It will play an essential role in the proof of the main result of this work. We recall that the domain of a function $f: X \rightarrow \mathbb{R} \cup\{+\infty\}$ is the set $\operatorname{dom} f:=f^{-1}(\mathbb{R})$.

Theorem 2 [4, See Theorem 4.4.18] Let $f, g: X \rightarrow \mathbb{R} \cup\{+\infty\}$ be convex and such that $0 \in$ int (dom $f-$ dom $g$ ), where int denotes interior. Then

$$
\inf _{x \in X}\{f(x)+g(x)\}=\sup _{x^{*} \in X^{*}}\left\{-f^{*}\left(x^{*}\right)-g^{*}\left(-x^{*}\right)\right\}
$$

and the supremum in (5) is attained if finite. 


\section{Main result}

We say that an lsc proper convex function $g: X \times X^{*} \rightarrow \mathbb{R} \cup\{+\infty\}$ is a twisted bigger conjugate function (TBC-function in short) [7, Definition 19.14] if

$$
-\left\langle x, x^{*}\right\rangle \leq g\left(x, x^{*}\right) \leq g^{*}\left(-x^{*},-x\right)
$$

for all $\left(x, x^{*}\right) \in X \times X^{*}$.

Example 3 The function $g: X \times X^{*} \rightarrow \mathbb{R} \cup\{+\infty\}$ defined by $g\left(x, x^{*}\right):=$ $f(x)+f^{*}\left(-x^{*}\right)$, where $f: X \rightarrow \mathbb{R} \cup\{+\infty\}$ is an lsc proper convex function, is a TBC-function. In particular, so is the function $g$ defined by $g\left(x, x^{*}\right):=$ $\frac{1}{p}\|x\|^{p}+\frac{1}{q}\left\|x^{*}\right\|_{*}^{q}$, where $p \geq 1$ and $\frac{1}{p}+\frac{1}{q}=1$.

The folowing proposition will be useful in the next section.

Proposition 4 Let $g: X \times X^{*} \rightarrow \mathbb{R} \cup\{+\infty\}$ be a non-negative TBC-function. Then

$$
\min _{\left(x, x^{*}\right) \in X \times X^{*}} g\left(x, x^{*}\right)=g(0,0)=0 .
$$

Proof. By (6) and the nonnegativity of $g$, we have

$$
0 \leq g(0,0) \leq g^{*}(0,0)=-\inf _{\left(x, x^{*}\right) \in X \times X^{*}} g\left(x, x^{*}\right) \leq 0 .
$$

Theorem 5 Let $X$ be reflexive, $T: X \rightrightarrows X^{*}$ be maximal monotone, and $g: X \times X^{*} \rightarrow \mathbb{R} \cup\{+\infty\}$ be a TBC-function. Consider the following conditions:

(i) $\operatorname{dom} F_{T}=X \times X^{*}$,

(ii) $\operatorname{dom} g=X \times X^{*}$.

If any one of them holds then

$$
F_{T}\left(x, x^{*}\right)-\left\langle x, x^{*}\right\rangle \geq \frac{1}{2} \inf _{\left(w, w^{*}\right) \in g p h T} g\left(w-x, w^{*}-x^{*}\right)
$$

for all $\left(x, x^{*}\right) \in X \times X^{*}$, with strict inequality whenever the infimum is not attained.

Proof. For $\left(x, x^{*}\right) \in X \times X^{*}$, consider the maximal monotone operator $L$ : $X \rightrightarrows X^{*}$ defined by $L(y):=T(y+x)-x^{*}$. Since

$$
g p h L=g p h T-\left(x, x^{*}\right),
$$


the functions $F_{L}$ and $g$ satisfy the assumptions of Theorem 2. Thus, since $F_{L}\left(x, x^{*}\right) \geq\left\langle x, x^{*}\right\rangle$ and $g\left(x, x^{*}\right) \geq-\left\langle x, x^{*}\right\rangle$ for every $\left(x, x^{*}\right) \in X \times X^{*}$, we have

$$
\begin{aligned}
0 & \leq \inf _{\left(x, x^{*}\right) \in X \times X^{*}}\left\{F_{L}\left(x, x^{*}\right)+g\left(x, x^{*}\right)\right\} \\
& =\sup _{\left(y^{*}, y\right) \in X^{*} \times X}\left\{-F_{L}^{*}\left(y^{*}, y\right)-g^{*}\left(-y^{*},-y\right)\right\} \\
& \leq \quad \sup _{\left(y^{*}, y\right) \in X^{*} \times X}\left\{-F_{L}^{*}\left(y^{*}, y\right)+\left\langle y, y^{*}\right\rangle\right\} \\
& \leq \sup _{\left(y^{*}, y\right) \in X^{*} \times X}\left\{-F_{L}\left(y, y^{*}\right)+\left\langle y, y^{*}\right\rangle\right\} \leq 0,
\end{aligned}
$$

the last two inequalities following from (4) and (3). Thereby

$$
\max _{\left(y^{*}, y\right) \in X^{*} \times X}\left\{-F_{L}^{*}\left(y^{*}, y\right)-g^{*}\left(-y^{*},-y\right)\right\}=0 .
$$

Thus, there exists $\left(y^{*}, y\right) \in X^{*} \times X$ such that $F_{L}^{*}\left(y^{*}, y\right)+g^{*}\left(-y^{*},-y\right)=0$. Вy (4), we obtain that

$$
F_{L}\left(y, y^{*}\right)+g^{*}\left(-y^{*},-y\right) \leq 0 .
$$

So, since $g$ is a TBC-function, we conclude that

$$
F_{L}\left(y, y^{*}\right)+g\left(y, y^{*}\right) \leq 0
$$

and thus, in view of the first inequality in (9), $F_{L}\left(y, y^{*}\right)+g\left(y, y^{*}\right)=0$ and $\left(y, y^{*}\right)$ is a minimizer of $F_{L}+g$.

Since

$$
0=F_{L}\left(y, y^{*}\right)+g\left(y, y^{*}\right) \geq F_{L}\left(y, y^{*}\right)-\left\langle y, y^{*}\right\rangle \geq 0
$$

we have

$$
-g\left(y, y^{*}\right)=F_{L}\left(y, y^{*}\right)=\left\langle y, y^{*}\right\rangle \text {. }
$$

Thus $\left(y, y^{*}\right) \in g p h L$ and, setting $\left(w, w^{*}\right):=\left(x+y, x^{*}+y^{*}\right)$, by (8) we obtain that $\left(w, w^{*}\right) \in g p h T$ and $-g\left(w-x, w^{*}-x^{*}\right)=\left\langle w-x, w^{*}-x^{*}\right\rangle$, and from Theorem 1 we conclude that

$$
\begin{aligned}
F_{T}\left(x, x^{*}\right)-\left\langle x, x^{*}\right\rangle & =F_{T}\left(w, w^{*}\right)-\left\langle w, w^{*}\right\rangle+F_{T}\left(x, x^{*}\right)-\left\langle x, x^{*}\right\rangle \\
& \geq-\frac{1}{2}\left\langle w-x, w^{*}-x^{*}\right\rangle=\frac{1}{2} g\left(w-x, w^{*}-x^{*}\right),
\end{aligned}
$$

which ends the proof.

Since the function $g$ of Example 3 is a TBC-function and its domain is $X \times X^{*}$, applying Theorem 5 to this case we obtain the following result:

Corollary 6 Let $X$ be reflexive, $T: X \rightrightarrows X^{*}$ be maximal monotone, and $f: X \rightarrow \mathbb{R} \cup\{+\infty\}$ be convex, proper and lsc. Consider the following conditions:

(i) $\operatorname{dom} F_{T}=X \times X^{*}$, 
(ii) $\operatorname{dom} f=X$ and dom $f^{*}=X^{*}$.

If any one of them holds then

$$
F_{T}\left(x, x^{*}\right)-\left\langle x, x^{*}\right\rangle \geq \frac{1}{2} \inf _{\left(w, w^{*}\right) \in g p h T}\left\{f(w-x)+f^{*}\left(x^{*}-w^{*}\right)\right\}
$$

for all $\left(x, x^{*}\right) \in X \times X^{*}$, with strict inequality whenever the infimum is not attained.

In particular, for every $p \geq 1$ and $q$ such that $\frac{1}{p}+\frac{1}{q}=1$, one has

$$
F_{T}\left(x, x^{*}\right)-\left\langle x, x^{*}\right\rangle \geq \frac{1}{2} \inf _{\left(w, w^{*}\right) \in g p h T}\left\{\frac{1}{p}\|w-x\|^{p}+\frac{1}{q}\left\|w^{*}-x^{*}\right\|_{*}^{q}\right\}
$$

for all $\left(x, x^{*}\right) \in X \times X^{*}$, with strict inequality whenever the infimum is not attained.

Concerning the condition $\operatorname{dom} f^{*}=X^{*}$ in (ii) of Corollary 6 , we recall that a sufficient (and necessary in the finite dimensional case) condition for it to hold is $f$ to be supercoercive [1, Theorem 3.4]:

$$
\lim _{\|x\| \longrightarrow+\infty} \frac{f(x)}{\|x\|}=+\infty .
$$

Setting $p=2$ in Corollary 6 , we obtain the following known result:

Corollary 7 (Strong Fitzpatrick Inequality) [8, Theorem 4] (see also [4, Theorem 9.7.2]). Let $X$ be reflexive and $T: X \rightrightarrows X^{*}$ be maximal monotone. Then

$$
\left.F_{T}\left(x, x^{*}\right)-\left\langle x, x^{*}\right\rangle \geq \frac{1}{4} \inf _{\left(w, w^{*}\right) \in g p h T}\left\{\|w-x\|^{2}+\| w^{*}-x^{*}\right) \|_{*}^{2}\right\}
$$

for all $\left(x, x^{*}\right) \in X \times X^{*}$.

\section{A new family of gap functions}

In this section, we shall consider the so called maximal monotone inclusion problem (MIP) [2,3]: Given a maximal monotone operator $T: X \rightrightarrows X^{*}$, find a point $x \in X$ such that

$$
0 \in T(x)
$$

The (possibly empty) solution set of MIP is $T^{-1}(0)=\{x \in X \mid 0 \in T(x)\}$.

A gap function for MIP is a function $\varphi: X \rightarrow \mathbb{R} \cup\{+\infty\}$ satisfying the following conditions:

i) $\varphi(x) \geq 0$ for all $x \in X$. 
ii) $\varphi(x)=0$ if and only if $x \in T^{-1}(0)$.

Thereby, the minimizers of $\varphi$ are exactly the elements in the solution set of MIP. Thus, we can reformulate MIP as the optimization problem consisting in minimizing $\varphi$. The following result has an immediate proof.

Proposition 8 (see [2, Theorem 2.1]) Let $\varphi$ be a gap function for MIP. If $M I P$ has a solution, then $\min _{x \in X} \varphi(x)=0$. Conversely, if $X$ is reflexive, $\inf _{x \in X} \varphi(x)=0$, and $\varphi$ is weakly lsc (in particular, if it is convex and lsc) and weakly coercive in the sense that

$$
\lim _{\|x\| \rightarrow \infty} \varphi(x)=+\infty
$$

then MIP has a solution.

In [2], Borwein and Dutta present a gap function $G_{T}$ for MIP, associated with the Fitzpatrick function $F_{T}$ of $T$. It is defined by $G_{T}(x):=F_{T}(x, 0)$, or, more explicitly,

$$
G_{T}(x)=\sup _{\left(y, y^{*}\right) \in g p h T}\left\langle x-y, y^{*}\right\rangle .
$$

It is easy to see that $G_{T}$ is indeed a gap function, and Borwein and Dutta found that it has good properties.

For $g: X \times X^{*} \rightarrow \mathbb{R} \cup\{+\infty\}$, we define $G_{T, g}: X \rightarrow \mathbb{R} \cup\{+\infty\}$ by

$$
G_{T, g}(x):=\frac{1}{2} \inf _{\left(w, w^{*}\right) \in g p h T} g\left(w-x, w^{*}\right) .
$$

Note that $G_{T, g}$ is convex whenever $g$ is convex, since in this case the function $\left(x, w, w^{*}\right) \mapsto g\left(w-x, w^{*}\right)$ is convex, jointly in its three arguments. Moreover, it is proper as long as $g$ is finite at some $\left(w, w^{*}\right) \in g p h T$.

The following proposition is an immediate consequence of Theorem 5 .

Proposition 9 Under the assumptions of Theorem 5, one has $G_{T} \geq G_{T, g}$, and hence $G_{T, g}$ is finite whenever $G_{T}$ is finite.

We refer to [2] for conditions on $T$ ensuring that $G_{T}$ is finite.

Definition 10 [6, Definition 10.1.1] Let $E$ be a metric space. An lsc function $\varphi: E \rightarrow \mathbb{R} \cup\{+\infty\}$ is said to be Tykhonov well-posed if it satisfies the following conditions:

(i) It has a unique global minimizer $\bar{x}$.

(ii) Every sequence $x_{n}$ such that $\varphi\left(x_{n}\right) \rightarrow \varphi(\bar{x})$ satisfies $x_{n} \rightarrow \bar{x}$.

Example 11 [6, Example 10.1.4] If $X$ is a finite dimensional vector space, then every lsc proper convex function $f: X \rightarrow \mathbb{R} \cup\{+\infty\}$ with a unique minimum point is Tykhonov well-posed. In particular, so is every lsc strictly convex function defined on $X$. 
Example 12 A function $f: X \rightarrow \mathbb{R} \cup\{+\infty\}$ is said to be strongly convex if there exists $\gamma>0$ such that, for every $x, y \in X$ and $\alpha, \beta \geq 0$ with $\alpha+\beta=1$, one has

$$
f(\alpha x+\beta y) \leq \alpha f(x)+\beta f(y)-\gamma \alpha \beta\|x-y\|^{2} .
$$

If $f$ is strongly convex and has a minimizer then it is Tykhonov well-posed. Indeed, it is well known that the minimizer $\bar{x}$, if it exists, must be unique. Let $x_{n}$ be a sequence satisfying $f\left(x_{n}\right) \rightarrow f(\bar{x})$. Setting $x:=x_{n}, y:=\bar{x}$ and $\alpha=\beta:=\frac{1}{2}$ in (10), we get

$$
\begin{aligned}
0 & \leq\left\|x_{n}-\bar{x}\right\| \leq \sqrt{\frac{2}{\gamma}\left(f\left(x_{n}\right)+f(\bar{x})-2 f\left(\frac{1}{2}\left(x_{n}+\bar{x}\right)\right)\right)} \\
& \leq \sqrt{\frac{2}{\gamma}\left(f\left(x_{n}\right)-f(\bar{x})\right)} \rightarrow 0,
\end{aligned}
$$

which shows that $x_{n} \rightarrow \bar{x}$.

For more examples and references about Tykhonov well-posedness, see $[5,6]$.

Theorem 13 Let $T: X \rightrightarrows X^{*}$ be maximal monotone and $g: X \times X^{*} \rightarrow \mathbb{R} \cup\{+\infty\}$ be a Tykhonov well-posed function satisfying (7). Then $G_{T, g}$ is a gap function for MIP.

Proof. Note that $G_{T, g}(x) \geq 0$ for all $x \in X$ since $g$ is non-negative. Moreover, if $G_{T, g}(x)=0$ for some $x \in X$ then there exist $\left(w_{n}, w_{n}^{*}\right) \subset g p h T$ such that $g\left(w_{n}-x, w_{n}^{*}\right) \longrightarrow 0$. Since $g$ is Tykhonov well-posed, from (7) it follows that $w_{n} \longrightarrow x$ and $w_{n}^{*} \longrightarrow 0$. Since $g p h T$ is closed, we deduce that $(x, 0) \in g p h T$, that is, $0 \in T(x)$. Conversely, if $0 \in T(x)$ then, using that $(x, 0) \in$ gph $T$ and (7), we obtain $0 \leq G_{T, g}(x) \leq g(0,0)=0$, concluding the proof.

Remark: For $x$ and $g$ as in Theorem (13), if there exist $\left(w, w^{*}\right) \in g p h T$ such that $g\left(w-x, w^{*}\right)=0$, then $G_{T, g}(x)=0$ and hence $x$ is a solution to MIP.

Corollary 14 Let $T: X \rightrightarrows X^{*}$ be maximal monotone and $g: X \times X^{*} \rightarrow \mathbb{R} \cup\{+\infty\}$ be a nonnegative Tykhonov well-posed TBC-function. Then $G_{T, g}$ is a gap function for MIP.

Proof. Combine Theorem 13 with Proposition 4.

\section{References}

[1] H. H. Bauschke, J. M. Borwein, P. L. Combettes. Essential smoothness, essential strict convexity, and Legendre functions in Banach spaces, Commun. Contemp. Math., 3 (2001), 615-647. 
[2] J. Borwein, J. Dutta. Maximal monotone inclusions and Fitzpatrick functions, special issue of the J Optim Theory Appl. on Nondifferentiable Optimization and Nonsmooth Analysis, dedicated to the memory of Vladimir Demyanov (accepted July 2015).

[3] J. M. Borwein, A. Lewis. Convex Analysis and Nonlinear Optmization: Theory and Examples, Springer, 2000.

[4] J. M. Borwein, J. D. Vanderwerff. Convex Functions: Constructions, Characterizations and Counterexamples, Cambridge University Press, 2010.

[5] A. L. Dontchev, T. Zolezzi. Well-Posed Optimization Problems, Lecture Notes in Mathematics 1543, Springer, 1993.

[6] R. Lucchetti. Convexity and well-posed problems, Springer, 2006.

[7] S. Simons. From Hahn-Banach to Monotonicity, Springer, 2008.

[8] M. D. Voisei, C. Zalinescu. Strongly-representable monotone operators., J. Convex Anal. 16 (2009), 1011-1033. 